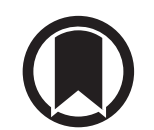

CrossMark

\title{
Increased focus on non-animal models for COVID-19 and non-COVID lung research
}

\author{
To the Editor:
}

Horizon Europe is a major stakeholder in the immediate future of lung research. As such, we believe it is worthwhile drawing the attention of the European Respiratory Journal readership to the recent publication by Hynes et al. [1] on Advanced Non-animal Models in Biomedical Research: Respiratory Tract Diseases. This report from the European Commission's Joint Research Centre EU Reference Laboratory (EURL ECVAM), and its accompanying dataset [2], identifies well-established current and emerging in vitro, in silico and ex vivo non-animal models for respiratory disease modelling, and reinforces the concept of "reduce, refine, or replace" animals used for scientific purposes. The $3 \mathrm{R}$ approach is not only embraced by the European Commission, but also the respiratory research community, as evidenced by many European Respiratory Society publications as well as frequent ERS Congress sessions and research seminars on the topic.

The EURL ECVAM study, prepared with the partnership of external experts in the field, involved a systematic review of peer-reviewed scientific literature. Over 21000 abstracts (11636 non-cancer and 9421 cancer) were scanned for relevant non-animal models of respiratory disease culminating in a knowledge base containing detailed descriptions of 284 non-animal models currently or recently being used for respiratory disease research. Briefly, the review shows that while simple models are still prominent and have their uses, in the past 5 years research focus has been shifting towards increasingly sophisticated bioengineering approaches that recapitulate human lung development, anatomy and physiology in vitro. Such novel approaches hold promise as blueprints for modelling human-relevant diseases and can be used not only to elucidate mechanism of disease, but also to aid in the development of new therapies and responses thereto. The findings from the study are freely available to download from the EURL ECVAM Collection in the JRC Data Catalogue, together with a JRC Technical Report that describes the review methodology and presents the main findings $[1,2]$. Ideally, this knowledge base will serve as a means to explore the strengths and limitations of both animal and non-animal models used in biomedical research; to stimulate healthy scientific debate; to challenge mindsets; and ultimately to pave the way for doing better science. Key conclusions of the work are summarised in table 1. Its overarching aim is to bridge methods and disciplines in order to improve biomedical science for the benefit of patients and society.

Respiratory diseases, including asthma, COPD, cystic fibrosis, pulmonary fibrosis and lung cancer represent a significant health burden to society. However, the majority of preclinical studies of new therapeutics for chronic lung diseases fail to identify effective treatments that are ultimately translated to the clinic, with over $90 \%$ of new drug programmes not progressing to market due to lack of efficacy or unexplained toxicity. This failure may be due in part to the use of preclinical models that do not fully recapitulate the complexity of human disease, are inherently hindered by interspecies differences, employ cells which are not specifically affected by the target disease in patients, or do not represent the genetic background of affected patients. Emerging non-animal preclinical models, such as lung-on-a-chip models, three-dimensional spheroid and organoid systems derived from primary and stem cell origins, and human tissue cultures such as precision cut lung slices, offer the opportunity to simulate human disease in vitro and ex vivo in a more robust and patient-specific manner [3-5]. Thereby, these novel investigative platforms provide important mechanistic information about respiratory diseases and identify targets for novel treatments.

@ERSpublications

A recent report from the European Commission highlights strategies for the development of safe and efficacious therapies for respiratory diseases, especially those that exploit non-animal methods and recapitulate the mechanistic basis of human disease https://bit.ly/2Vk3xfA

Cite this article as: Gribaldo L, Hurley K, Hiemstra P, et al. Increased focus on non-animal models for COVID-19 and non-COVID lung research. Eur Respir J 2021; 57: 2004267 [https://doi.org/10.1183/ 13993003.04267-2020]. 
TABLE 1 Key summary points related to uptake, development and standardisation of non-animal models for respiratory disease research

Standard operating procedures for model generation and testing are essential

The specific purpose of any model must be clearly defined

Realistic lung-on-a-chip models will become more widespread, especially for biomarker studies

Plastic substrates in any model are not representative of human tissue and should be replaced

To improve organoid models more effective cross-talk between oncology and respiratory fields is needed

Lessons learned from lung cancer organoid studies could be equally valid for the wider respiratory disease field

Pioneering functional organoid studies in cystic fibrosis have relevance for other inflammatory and infectious lung diseases

Precision cut lung slice (PCLS) is the most representative human model in terms of cell types, tissue architecture and physiological response, notwithstanding difficulties in obtaining sample material

Adapted from [1].

The current coronavirus disease 2019 (COVID-19) crisis also highlights the need for better patient- and disease-relevant lung models. In the relatively short period since the identification of SARS-CoV-2 as the causative virus of COVID-19, important progress has been made in advancing our knowledge about the disease using in vitro culture methods and in silico approaches. A variety of approaches have been used for a range of purposes, including the study of epithelial cell tropism of the virus [6], effectivity of antiviral drugs [7], and application of organoids [8] and lung-on-chip models for COVID-19 modelling [9]. In addition to using primary lung epithelial cells isolated from lung tissue, human induced pluripotent stem cell-derived alveolar type 2 cells have been used to obtain insight into the transcriptional response of these cells to infection, and their potential use for drug testing [10]. Whereas these examples used epithelial culture models, other studies have used cells from other organs to delineate their involvement in COVID-19. Careful matching of the research question to the culture model used and an awareness of the limitations intrinsic to any model remains essential.

In conclusion, this report from the European Commission highlights research strategies for the development of safe and efficacious new therapies for well-known and emerging respiratory diseases especially those that exploit non-animal methods and recapitulate the mechanistic basis of human disease. Such approaches are widely welcomed and in due course are likely to outnumber and outperform animal studies.

Laura Gribaldo ${ }^{1}$, Killian Hurley ${ }^{2}$, Pieter Hiemstra $\oplus^{3}$ and Catherine Greene $\odot^{4}$

${ }^{1}$ European Commission, Joint Research Center (JRC), Ispra, Italy. ${ }^{2}$ Dept of Medicine, Royal College of Surgeons in Ireland, Dublin, Ireland. ${ }^{3}$ Dept of Pulmonology, Leiden University Medical Center, Leiden, The Netherlands. ${ }^{4}$ Dept of Clinical Microbiology, Royal College of Surgeons in Ireland, Dublin, Ireland.

Correspondence: Catherine Greene, Royal College of Surgeons in Ireland, Dept of Clinical Microbiology, RCSI Education and Research Centre, Beaumont Hospital, Dublin 9, Ireland. E-mail: CMGreene@rcsi.ie

Received: 19 Nov 2020 | Accepted: 20 Nov 2020

Conflict of interest: L. Gribaldo has nothing to disclose. K. Hurley has nothing to disclose. P. Hiemstra reports grants from the Dutch Society for the Replacement of Animal Testing, outside the submitted work. C. Greene has nothing to disclose.

\section{References}

1 Hynes J, Marshall L, Adcock I, et al. Advanced Non-animal Models in Biomedical Research: Respiratory Tract Diseases. EUR 30334 EN. Publications Office of the European Union, Luxembourg, 2020.

2 European Commission. Joint Research Centre Data Catalogue. EURL ECVAM Review of Non-animal Models in Biomedical Research: Respiratory Tract Diseases. https://data.jrc.ec.europa.eu/dataset/176d71e6-5082-4b29-8472b719f6bda323

3 Bonniaud P, Fabre A, Frossard N, et al. Optimising experimental research in respiratory diseases: an ERS statement. Eur Respir J 2018; 51: 1702133.

$4 \quad$ Hiemstra PS, Tetley TD, Janes SM. Airway and alveolar epithelial cells in culture. Eur Respir J 2019; 54: 1900742.

5 Alsafadi HN, Uhl FE, Pineda RH, et al. Applications and approaches for three-dimensional precision-cut lung slices. Disease modeling and drug discovery. Am J Respir Cell Mol Biol 2020; 62: 681-691.

6 Hou YJ, Okuda K, Edwards CE, et al. SARS-CoV-2 reverse genetics reveals a variable infection gradient in the respiratory tract. Cell 2020; 182: 429-446.e14. 
7 Hoffmann M, Kleine-Weber H, Schroeder S, et al. SARS-CoV-2 cell entry depends on ACE2 and TMPRSS2 and is blocked by a clinically proven protease inhibitor. Cell 2020; 181: 271-280.e8.

8 Lamers MM, Beumer J, van der Vaart J, et al. SARS-CoV-2 productively infects human gut enterocytes. Science 2020; 69: 50-54.

9 Tang H, Abouleila Y, Si L, et al. Human organs-on-chips for virology. Trends Microbiol 2020; 28: 934-946.

10 Huang J, Hume AJ, Abo KM, et al. SARS-CoV-2 infection of pluripotent stem cell-derived human lung alveolar type 2 cells elicits a rapid epithelial-intrinsic inflammatory response. Cell Stem Cell 2020; 27: 962-973. 